\title{
Operation and Performance of the ATLAS Muon Spectrometer Databases during 2011-12 Data Taking
}

\author{
Monica Verducci* \\ University of Washington, Department of Physics, FM-15, Seattle WA 98195, USA \\ E-mail: monica.verducci@cern.ch
}

\begin{abstract}
The size and complexity of the ATLAS experiment at the Large Hadron Collider, including its Muon Spectrometer, raise unprecedented challenges in terms of operation, software model and data management. One of the challenging tasks is the storage of non-event data produced by the calibration and alignment stream processes and by online and offline monitoring frameworks, which can unveil problems in the detector hardware and in the data processing chain. During 2011 and 2012 data taking, the software model and data processing enabled high quality track resolution as a better understanding of the detector performance was developed using the most reliable detector simulation and reconstruction. This work summarises the various aspects of the Muon Spectrometer Databases, with particular emphasis given to the Conditions Databases and their usage in the data analysis.
\end{abstract}

The European Physical Society Conference on High Energy Physics -EPS-HEP2013

18-24 July 2013

Stockholm, Sweden

${ }^{*}$ Speaker. 


\section{Introduction}

The ATLAS Muon Spectrometer (MS) is the outer part of the ATLAS detector and is designed to detect charged particles exiting the barrel and end-cap calorimeters and to measure their momentum in the pseudorapidity range $|\eta|<2.7$. It is also designed to trigger on these particles in the region $|\eta|<2.4$, [1]. The quality of the muon measurement has been one of the guiding design criteria for the ATLAS experiment, the performance goal is a stand-alone transverse momentum resolution of approximately $10 \%$ for $1 \mathrm{TeV}$ tracks, which translates into a sagitta along the $\mathrm{z}$ (beam) axis of about $500 \mu \mathrm{m}$, to be measured with a resolution of $60 \mu \mathrm{m}$. This concern is reflected by the choice of the main components of the muon spectrometer: a system of three large superconducting air-core toroid magnets, precision tracking detectors with $60 \mu \mathrm{m}$ intrinsic resolution (Monitored Drift Tube chambers, MDT, and the Cathode-Strip Chambers, CSC), and a powerful dedicated trigger system (Resistive Plate Chambers, RPC and Thin Gap Chambers, TGC). The monitoring and the storage in DataBases (DB) of all the detector run parameters are additionally mandatory to achieve such a challenging performance.

\section{The Muon Databases}

The muon system accesses to several databases, which hold very different types of data:

- Configuration Database. This database stores all the data needed at the start of the run, including sub-detector hardware and software configuration. It registers the different configurations available (cosmics, physics,...) for each sub-detector.

- Calibration Database. This database holds all the calibration constants produced by calibration jobs within the offline software. Single muon tracks from the second level trigger, are streamed to Tier2 farms, where the new calibration constants are produced and written to a local Calibration Database [2].

- Conditions Database. These databases store non-event detector data, which can vary with time and exist in different versions. These data can be produced by both offline and online environments and, in general, are used for diagnostic by detector experts.

\subsection{The Muon Conditions Database}

The conditions database will store all the parameters describing run conditions and logging, all the data which will be accessed offline, i.e. by the reconstruction or analysis software. Conditions data varies with time, and is usually characterized by an "interval of validity"(IoV). It includes data archived from the ATLAS detector control system (DCS), online book-keeping data, online and offline calibration and alignment data, and monitoring data characterizing the performance of the detector during any particular period of time [3].

The ATLAS conditions database is based on Oracle DB and it is implemented using COOL technology [4]. COOL, an LCG product is a library to manage conditions data in terms of IoV, versions and tags. Several special-purpose higher level interfaces are also being developed, including the Condition Database Interface (CDI) for archiving Information System (IS) data to COOL, the 
PVSS to COOL interface for archiving Detector Control System (DCS) data, specialized interfaces for saving monitoring data, and the IOVService, a software interface between the COOL DB and the offline algorithms.

\subsubsection{The Alignment Example}

The alignment procedure requires for each station three angles, three shifts and eight deformation parameters, provided by a sophisticated optical alignment system, based on laser beams and CCD cameras, and offline alignment procedures using muon tracks for overlapping regions. This system aligns the MS with respect to Inner Tracker at the level of few 100 microns. In total, the MS system has 3 large subsystems (Barrel +2 Endcaps), this means 1200 MDT precision chambers each described by 6 positional parameters and 11 chamber internal deformation parameters, giving a total of $\sim 21000$ degree of freedom. The conversion from optical sensors measurements taken from Oracle DB into chamber position and deformation parameters (via a fit) is stored to Oracle DB (ATLR). All input/output with the DB is performed via a Java application deployed in a J2EE Application Server. The Alignment corrections are migrated to Condition DB via python interfaces for further usage by track reconstruction programs. The Alignment loop has been tested in 2009 and it worked perfectly during the three years of data-taking.

\subsection{The Calibration Databases}

The Calibration Database stores all the constants needed to fast calibrate the 350k drift tubes of the MDT. To obtain high statistics for the calibrations we extract single muon tracks from the second level trigger, where the preselection of data by the first level trigger allows the selection of only hits from isolated muon tracks. The collected data are then streamed using ATLAS grid data transfer to remote Tier 2 farms dedicated to detector calibration. The calibration stream event rate is about 10X the rate of single muons in regular ATLAS data and is processed in 36 hours. The calibration centers process the data with a program called the Local Calibration Data Splitter which divides the data into 204 regions for processing a computer farm. The final new calibration constants are then written to a local calibration database ( $\sim \mathrm{M}$ of data per run) which is used to fill the ATLAS Conditions DB. The entire loop from the close of data-taking to the loading of new calibration constants in the COOL DB is completed in under 36 hours. This calibration procedure has run very well for past three years on the ATLAS experiment at the Large Hadron Collider.

\section{References}

[1] ATLAS Muon Collaboration, "The ATLAS Muon Spectrometer Technical Design Report", CERN-LHCC/97-22 [Online], May 311997.

[2] E.Pasqualucci, et al. "Muon detector calibration in the ATLAS experiment: data extraction and distribution", in Proceedings of CHEP06, Mumbai, India, 13-17 Feb. 2006

[3] M. Verducci, "ATLAS Conditions Database and Calibration Streams", Nuclear Physics B (Proc.Suppl.) 172 (2007) 250-252

[4] A. Valassi, COOL web page, Available at http://lcgapp.cern.ch/project/CondDB/ 